\title{
An Integrated Inventory Model for Vendor and Buyer
}

\author{
Zhiguang Zhang \\ Department of Mathematics, Dezhou University \\ Dezhou 253023, China \\ E-mail: zhiguangzhang@126.com
}

\begin{abstract}
An integrated inventory model for vendor and buyer is presented in this paper. Based on the integrated expected total relevant costs of both buyer and vendor, we obtain the optimal values of reorder point, order quantity, and number of shipments. Numerical example shows the model is effective, and it is also used to analyze the savings in joint total cost over individually derived policies.
\end{abstract}

Keywords: Inventory, Vendor, Buyer

\section{Introduction}

With the increased competition in today's global markets, companies are forced to closely work in partnership with their customers and suppliers. More and more individual businesses work together as a supply chain and no longer compete as solely independent entities (Min H et al.2002, Lambert DM et al.2000). With the growing focus on supply chain management, many companies are recognizing that inventories across the entire supply chain can be more efficiently managed through better coordination of the supply chain. The coordination of the supply chain is very important, otherwise if no such coordination exists, the vendor and the buyer will act independently to minimize their costs. If each stage of the supply chain only optimizes its local objective without considering the impact on the complete chain, lack of coordination results that total supply chain profits are less than what could be achieved through coordination (Stadtler H 2005, Goetschalckx M et al.2002). Each stage of the supply chain, in trying to optimize its local objective, takes actions that end up hurting the performance of the entire supply chain. A manager can use lot size-based quantity discounts, buyback contracts or quantity flexibility contracts and etc to achieve coordination.

This paper considers a supply chain consisting of one vendor and one buyer. The buyer orders lots to the vendor. The vendor produces the requested product in lots and ships each lot to the buyer in batches. In traditional inventory systems, the optimal ordering and shipment policies for vendor and buyer are managed independently. As a result, the lot size of purchaser may not result in an optimal policy for the vendor and vice versa. To overcome this disadvantage, researchers have studied the integrated inventory model of vendor and buyer, where the joint total relevant cost for the purchaser as well as the vendor has been optimized. On the other hand, it will be more effective to determine the ordering and shipment policies based on their integrated total cost function, rather than using the buyer's or the supplier's individual cost functions. The integrated inventory problem has received a lot of attention in recent years as it is one of the building blocks for the wider supply chain (Ben-Daya M et al.2008, Goyal Sk 1977, Hill RM 1999).

Although large number of researches extending different dimensions of the problems, however most of them are limited to deterministic conditions. In recent years, increasing attention is given to the development of non-deterministic integrated inventory models. In this paper, we develop an integrated inventory system consisting of a single vendor and single buyer, where the lot from the vendor is transferred to the buyer in equal-sized batches. The supply lead-time between vendor and buyer is considered to be stochastic. Moreover, the model is extended to the situation in which shortage is allowed.

This paper is organized as follows. In section 2, the notations and assumptions are introduced. In section 3 , independent optimization models of buyer and vendor are given. Section 4 presents the integrated model and an algorithm is developed to find the optimal solution of the integrated model. A numerical example is showed in section 5 .

\section{Notations and Assumptions}

\section{1 notations}

$Q$ : buyer's order quantity;

$D$ : demand per unit time;

$h_{v}$ : inventory holding cost for the vendor per unit per unit time; 
$h_{b}$ : inventory holding cost for the buyer per unit per unit time;

$r$ : buyer's reorder point;

$A_{1}$ : buyer's ordering cost;

$A_{2}$ : vendor's setup cost;

$s:$ shortage cost for the buyer per unit per time;

$n$ : number of shipments;

$L$ : lead-time to replenish the buyer's order.

$T$ : buyer's cycle time;

\subsection{Assumptions}

The demand rate is deterministic and constant. The supply chain is consisted of one vendor and one buyer. The vendor's production rate is infinite. The buyer orders a lot of size $Q$ when the inventory reaches the reorder point $r$. Inventory is continuously reviewed. Shortages are allowed and completely backordered. Lead time to replenish the buyer's order is stochastic and follows a uniform distribution with parameters $a$ and $b$.

\section{Independent Optimization Models}

When the vendor and buyer don't choose to cooperate, we give the buyer's and the vendor's optimal models and its solution respectively. Then we present the integrated inventory model and compare the independent optimal policies and the integrated policy.

\section{1 buyer's optimization model}

In order to get the optimal ordering policy of the buyer, we need to obtain the buyer expected total cost per unit time $C_{b}(r, Q)$. We assume that the orders do not cross in time and the order quantity received by the buyer is equal to or greater than the maximum demand during lead time. The lead time is assumed to be uniformly distributed, then

$$
L \square U[a, b], f(l)=\frac{1}{b-a}, a \leq l \leq b .
$$

During an order cycle, the buyer expected total cost is calculated by multiplying the inventory carried and shortages incurred during each cycle time and taking the expectation over the relevant limits for the lead time. then we obtain the expected total cost per unit time, dividing these costs by the length of the buyer order cycle, $Q / D$. When $r \leq D b$, there will be any shortages. But shortages will not occur if $r>D b$.

Thus, we obtain the following expected total cost per time for $r \leq D b$ :

$$
\begin{aligned}
C_{b 1}(r, Q)=\frac{D A_{1}}{Q} & +\frac{D h_{b}}{Q} \int_{a}^{\frac{r}{D}}\left(r-\frac{D l}{2}\right) \frac{l}{b-a} d l+\frac{r h_{b}}{Q} \int_{a}^{b}(Q-D l) \frac{1}{b-a} d l \\
& +\frac{h_{b}}{2 Q} \int_{a}^{b}(Q-D l) \frac{1}{b-a} d l+\frac{r^{2} h_{b}}{2 Q} \int_{\frac{r}{D}}^{b} \frac{1}{b-a} d l+\frac{s}{2 Q} \int_{\frac{r}{D}}^{b}(D l-r)^{2} \frac{1}{b-a} d l \\
& =\frac{D A_{1}}{Q}+h_{b} r+\frac{h_{b}\left(2 r^{3}-3 r D^{2} a^{2}+D^{3} a^{3}\right)}{6 D Q(b-a)}-\frac{h_{b} r D(b+a)}{2 Q}+\frac{h_{b}(Q-D b-D a)}{2} \\
+ & \frac{h_{b} D^{2}\left(b^{2}+a b+a^{2}\right)}{6 Q}+\frac{h_{b} r^{2}(D b-r)}{2 D Q(b-a)}+\frac{s(D b-r)^{3}}{6 D Q(b-a)}
\end{aligned}
$$

We obtain the following expected total cost per time for $r>D b$ :

$$
\begin{aligned}
& C_{b 2}(r, Q)=\frac{D A_{1}}{Q}+\frac{D h_{b}}{Q} \int_{a}^{\frac{r}{D}}\left(r-\frac{D l}{2}\right) \frac{l}{b-a} d l+\frac{r h_{b}}{Q} \int_{a}^{b}(Q-D l) \frac{1}{b-a} d l+\frac{h_{b}}{2 Q} \int_{a}^{b}(Q-D l)^{2} \frac{1}{b-a} d l \\
& =\frac{D A_{1}}{Q}+h_{b} r-\frac{h_{b} D(b+a)}{2}+\frac{h_{b} Q}{2}
\end{aligned}
$$


Thus, the buyer expected total cost per time unit is

$$
C_{b}(r, Q)=\min \left\{C_{b 1}(r, Q), C_{b 2}(r, Q)\right\} .
$$

Theorem $C_{b 1}(r, Q), C_{b 2}(r, Q)$ are convex.

Proof for the case $r \leq D b$, we obtain the Hessian matrixes $H$ of $C_{b 1}(r, Q)$ as follows

$$
H=\left(\begin{array}{cc}
\frac{\left(h_{b}+s\right)(D b-r)}{D Q(b-a)} & \frac{6 D^{2} A_{1}(b-a)+\left(h_{b}+s\right)(D b-r)^{3}}{3 D Q^{3}(b-a)} \\
\frac{6 D^{2} A_{1}(b-a)+\left(h_{b}+s\right)(D b-r)^{3}}{3 D Q^{3}(b-a)} & \frac{\left(h_{b}+s\right)(D b-r)^{2}}{2 D Q^{2}(b-a)}
\end{array}\right)
$$

Hessian determinants can be obtained as follows $\quad\left|H_{1}\right| \geq 0,|H| \geq 0$.

Therefore the Hessian matrix is positive and $C_{b 1}(r, Q)$ is convex. By the same method, we prove $C_{b 2}(r, Q)$ is also convex.

The proof is completed.

Since the buyer expected total cost per time unit is convex, the local optimum solution is global optimum solution. Therefore we use a numerical procedure to find the optimal values of the nonlinear programming problems.

3.2 vendor's optimization model

When the purchaser order quantity $Q$ is adopted, the orders are received by the vendor at intervals $T$. Then the vendor expected total cost per time is

$C_{v}(n)=\frac{D A_{2}}{n Q}+\frac{(n-1) Q h_{v}}{2}$

We can easily prove the is convex and obtain the following optimality conditions on $n^{*}$

$n^{*}\left(n^{*}-1\right) \leq \frac{2 D A_{2}}{h_{v} Q} \leq n^{*}\left(n^{*}+1\right)$

When the vendor and buyer are free to choose their own ordering policy $n,(r, Q)$, the individually derived expected total cost $T C(Q, r, n)=C_{b}(r, Q)+C_{v}(n)$ is equal to the summation of buyer's and vendor's sub-optimal expected costs.

\section{Integrated Vendor-Buyer Optimization Model}

Suppose that the vendor and buyer decide to cooperate and follow the jointly optimal policy. Then the joint expected total cost of the buyer and the vendor per time unit for $r \leq D b$ is

$$
\begin{aligned}
& J C_{1}(Q, r, n)=\frac{D\left(n A_{1}+A_{2}\right)}{n Q}+\frac{Q\left(h_{b}+(n-1) h_{v}\right)}{2}+h_{b} r+\frac{h_{b}\left(2 r^{3}-3 r D^{2} a^{2}+D^{3} a^{3}\right)}{6 D Q(b-a)} \\
& -\frac{h_{b} D(b+a)(r+Q)}{2 Q}+\frac{h_{b} D^{2}\left(b^{2}+a b+a^{2}\right)}{6 Q}+\frac{h_{b} r^{2}(D b-r)}{2 D Q(b-a)}+\frac{s(D b-r)^{3}}{6 D Q(b-a)} .
\end{aligned}
$$

We obtain the following expressions for $r>D b$ :

$J C_{2}(Q, r, n)=\frac{D\left(n A_{1}+A_{2}\right)}{n Q}+\frac{Q\left(h_{b}+(n-1) h_{v}\right)}{2}+h_{b} r-\frac{h_{b} D(b+a)}{2}$.

Then we can obtain the joint expected total cost per time

$J C(Q, r, n)=\min \left\{J C_{1}(Q, r, n), J C_{2}(Q, r, n)\right\}$. 
We can easily prove that $J C_{1}(Q, r, n)$ and $J C_{2}(Q, r, n)$ are convex. The optimal solution of the nonlinear programming can be gotten using LINGO 9.0.

\section{Numerical Experiments}

We consider an example with the following data: $D=1000 /$ year, $A_{1}=\$ 25 /$ order,$A_{2}=\$ 400 /$ setup , $h_{b}=\$ 10 /$ unit / year, $h_{v}=\$ 8 /$ unit / year,$s=\$ 30 /$ unit / year $, a=0, b=35$. For the independent optimization model, the optimal values of $r, Q, n$ and expected total cost per time are 27.7, 97.0, 3 and 2918.1. The corresponding values for the integrated optimization model are $0,311.9,1$ and 2639.2 , the total saving cost is $9.56 \%$ and it should be shared by the vendor and the buyer in some equitable manner.

\section{References}

Ben-Daya M, Darwish M, Ertogral K. (2008). The joint economic lot sizing problem: review and extensions. Eur J Oper Res vol.185, pp.726-742.

Goetschalckx M, Vidal CJ, Dogan K. (2002). Modeling and design of global logistics systems: a review of integrated strategic and tactical models and design algorithms. Eur J Oper Res vol.143(1), pp. 1-18.

Goyal Sk. (1977). An integrated inventory model for a single supplier-single customer problem. Int J Prod Res vol15(1), pp.107-111.

Hill RM. (1999). The optimal production and shipment policy for the single-vendor single-buyer integrated production-inventory model. Int J Prod Res vol.37, pp.2463-2475.

Lambert DM, Cooper MC. (2000). Issues in supply chain management. Ind Mark Manage vol.29(1), pp. 65-83.

Min H, Zhou GG. (2002). Supply chain modeling: past, present and future. Comput Ind Eng vol. 43(1-2), pp. 231-249.

Stadtler H. (2005). Supply chain management and advanced planning-basics, overview and challenges. Eur $J$ Oper Res vol.163(3), pp. 575-588.

Y. Salama. (2000). Optimal control of a simple manufacturing system with restarting costs, Operations Research Letters, vol.26, pp.9-16.

Zhiguang Zhang. (2010). optimal solutions of production inventory control problem, Mordren Applied Science, vol.4, pp.90-94. 